\title{
Predictor - Corrector Methods of High Order for Numerical Integration of Initial Value Problems
}

\author{
Abdulrahman Ndanusa \\ Department of Mathematics \\ Federal University of Technology, \\ Minna, Nigeria \\ as.ndanusa@futminna.edu.ng
}

\author{
Fatima Umar Tafida \\ Department of Mathematics \\ Federal University of Technology, \\ Minna, Nigeria \\ fatimiumartafida@yahoo.com
}

\begin{abstract}
Two tenth order implicit linear multistep methods are derived after applying appropriate order conditions to the Taylor series approach in the derivation of linear multistep methods. Each of the derived schemes is further combined with an Adams - Bashforth scheme of order ten to form two separate predictorcorrector pairs for numerical integration of initial value problems of ordinary differential equations. A tenth order Runge - Kutta method is further employed in order to generate the necessary starting values typical of linear multistep methods. The derived schemes are proven to be convergent by satisfying both consistency and zero - stability requirements. Numerical examples are further carried out to ascertain their efficiency and effectiveness.
\end{abstract}

Keywords: Predictor - corrector method, Linear multistep method, Runge - kutta method, Stability, Adams Bashforth method.

\section{INTRODUCTION}

A great many problems in science, engineering and real life applications, more often than not, are modeled as systems of first-order differential equations in ${ }^{n}$ dependent variables $y_{1}, y_{2, \ldots}, y_{n}$ thus:

$$
\left.\begin{array}{c}
y_{1}^{s}=f_{1}\left(x_{1}, y_{1}, y_{2}, \ldots, y_{n}\right) \\
y_{2}^{s}=f_{2}\left(x_{1}, y_{1}, y_{2}, \ldots, y_{n}\right) \\
\cdot \\
\cdot \\
y_{n}^{s}=f_{1}\left(x_{1}, y_{1}, y_{2}, \ldots, y_{n}\right)
\end{array}\right\}
$$

An alternative form of writing the initial value problem (IVP) (1) and (2) is

$$
y^{\prime}=f(x, y), \quad y\left(x_{0}\right)=y_{0}
$$

where, $\boldsymbol{y}=\left[y_{1}, y_{2}, \ldots, y_{n}\right]^{T}, \boldsymbol{f}=\left[f_{1}, f_{2}, \ldots, f_{n}\right]^{T}=\boldsymbol{f}\left(x_{,} \boldsymbol{y}\right), \boldsymbol{y}_{0}=\left[y_{10}, y_{20}, \ldots, y_{n 0}\right]^{T}$.

A numerical solution of the IVP (3) consists of a sequence of values $\left\{y_{n}\right\}$ which estimates the solution (3) on the distinct point set ${ }^{\left\{x_{n}\right\}}$. Suppose a ${ }^{k-\text { step }}$ linear multistep method (LMM) is employed to solve (3), then each step of the solution process involves the equation

$$
y_{n+k}+\sum_{j=0}^{k-1} \alpha_{j} y_{n+j}=h \beta_{k} f\left(x_{n+k^{n}} y_{n+k}\right)+h \sum_{j=0}^{k-1} \beta_{j} f_{n+j}
$$


where $y_{n+j^{j}} f_{n+j^{j}} j=0,1_{p} \cdots, k-1$ are known [1]. We proceed by using a separate explicit linear multistep method to estimate an approximation to ${ }^{y_{n+k}}$ and use this predicted value on the right hand side of (4) in the evaluation of $f\left(x_{n+k^{s}} y_{n+k}\right)$. The implicit method is then used for a predetermined number of steps. The explicit method is called the predictor and the implicit method is called the corrector. Popular predictor - corrector methods in use include the Milne's method [2], Hamming's method [3], Klopfenstein - Millman algorithm [4], Crane - Klopfenstein algorithm [5], Krogh's method [6] and Ndanusa and Adeboye's method [7].

In this paper, we analyze the development of two implicit linear multistep methods of order ten which are combined with a tenth order Adams - Bashforth method, to form two predictor - corrector methods for the numerical integration of initial value problems.

\section{METHODS}

\subsection{Derivation of the LMMs of Order Ten}

Since it is required that the corrector methods be of order 10 , the step number of the methods, ${ }^{k}$, has to be 8 . Thus, the first characteristic polynomial $\rho(\xi)$ takes the form

$$
\begin{aligned}
\rho(\xi)=\xi^{8}- & (2 a+2 b+2 c) \xi^{7}+(2+4 b c+4 a c+4 a b) \xi^{6} \\
+ & (-2 b-2 c-4 a-8 a b c-2 a) \xi^{5}-(2 a-4 a-8 a b c-2 c-2 b) \xi^{3} \\
& -(-4 a b-4 a c-a-2 b c) \xi^{2}+(2 a+2 b+2 c) \xi-1
\end{aligned}
$$

where

$$
\left.\begin{array}{c}
\alpha_{8}=+1, \quad \alpha_{7}=-2(a+b+c), \quad \alpha_{6}=2(1+2 a b+2 a c+2 b c) \\
\alpha_{5}=-2(a+b+c+4 a b c), \quad \alpha_{4}=0, \quad \alpha_{3}=2(a+b+c+4 a b c) \\
\alpha_{2}=-2(1+2 a b+2 a c+2 b c), \quad \alpha_{1}=2(a+b+c), \quad \alpha_{0}=-1
\end{array}\right\}
$$

Therefore, the order conditions are expressed as follows

$$
\begin{aligned}
& D_{0}=\alpha_{0}+\alpha_{1}+\alpha_{2}+\alpha_{3}+\alpha_{4}+\alpha_{5}+\alpha_{6}+\alpha_{7}+\alpha_{8} \\
& D_{1}=\left[-r \alpha_{0}+(1-r) \alpha_{1}+(2-r) \alpha_{2}+\cdots+(7-r) \alpha_{7}+(8-r) \alpha_{8}\right] \\
& -\left[\beta_{0}+\beta_{1}+\beta_{2}+\beta_{3}+\beta_{4}+\beta_{5}+\beta_{6}+\beta_{7}+\beta_{8}\right] \\
& D_{2}=\frac{1}{2 !}\left[(-r)^{2} \alpha_{0}+(1-r)^{2} \alpha_{1}+(2-r)^{2} \alpha_{2}+\cdots+(7-r)^{2} \alpha_{7}+(8-r)^{2} \alpha_{8}\right] \\
& -\left[-r \beta_{0}+(1-r) \beta_{1}+(2-r) \beta_{2}+\cdots+(7-r) \beta_{7}+(8-r) \beta_{8}\right] \\
& D_{3}=\frac{1}{3 !}\left[(-r)^{3} \alpha_{0}+(1-r)^{3} \alpha_{1}+(2-r)^{3} \alpha_{2}+\cdots+(7-r)^{3} \alpha_{7}+(8-r)^{3} \alpha_{8}\right] \\
& -\frac{1}{2 !}\left[-r^{2} \beta_{0}+(1-r)^{2} \beta_{1}+(2-r)^{2} \beta_{2}+\cdots+(7-r)^{2} \beta_{7}+(8-r)^{2} \beta_{8}\right] \\
& D_{4}=\frac{1}{4 !}\left[(-r)^{4} \alpha_{0}+(1-r)^{4} \alpha_{1}+(2-r)^{4} \alpha_{2}+\cdots++(7-r)^{4} \alpha_{7}+(8-r)^{4} \alpha_{8}\right] \\
& -\frac{1}{3 !}\left[-r^{3} \beta_{0}+(1-r)^{3} \beta_{1}+(2-r)^{3} \beta_{2}+\cdots++(7-r)^{3} \beta_{7}+(8-r)^{3} \beta_{8}\right] \\
& D_{5}=\frac{1}{5 !}\left[(-r)^{5} \alpha_{0}+(1-r)^{5} \alpha_{1}+(2-r)^{5} \alpha_{2}+\cdots+(7-r)^{5} \alpha_{7}+(8-r)^{5} \alpha_{8}\right]
\end{aligned}
$$




$$
\begin{aligned}
& -\frac{1}{4 !}\left[-r^{4} \beta_{0}+(1-r)^{4} \beta_{1}+(2-r)^{4} \beta_{2}+\cdots+(7-r)^{4} \beta_{7}+(8-r)^{4} \beta_{8}\right] \\
D_{6}= & \frac{1}{6 !}\left[(-r)^{6} \alpha_{0}+(1-r)^{6} \alpha_{1}+(2-r)^{6} \alpha_{2}+\cdots+(7-r)^{6} \alpha_{7}+(8-r)^{6} \alpha_{8}\right] \\
& \quad-\frac{1}{5 !}\left[-r^{5} \beta_{0}+(1-r)^{5} \beta_{1}+(2-r)^{5} \beta_{2}+\cdots+(7-r)^{5} \beta_{7}+(8-r)^{5} \beta_{8}\right] \\
D_{7}= & \frac{1}{7 !}\left[(-r)^{7} \alpha_{0}+(1-r)^{7} \alpha_{1}+(2-r)^{7} \alpha_{2}+\cdots+(7-r)^{7} \alpha_{7}+(8-r)^{7} \alpha_{8}\right] \\
& -\frac{1}{6 !}\left[-r^{6} \beta_{0}+(1-r)^{6} \beta_{1}+(2-r)^{6} \beta_{2}+\cdots+(7-r)^{6} \beta_{7}+(8-r)^{6} \beta_{8}\right] \\
D_{8}= & \frac{1}{8 !}\left[(-r)^{8} \alpha_{0}+(1-r)^{8} \alpha_{1}+(2-r)^{8} \alpha_{2}+\cdots+(7-r)^{8} \alpha_{7}+(8-r)^{8} \alpha_{8}\right] \\
& -\frac{1}{7 !}\left[-r^{7} \beta_{0}+(1-r)^{7} \beta_{1}+(2-r)^{7} \beta_{2}+\cdots+(7-r)^{7} \beta_{7}+(8-r)^{7} \beta_{8}\right] \\
D_{9}= & \frac{1}{9 !}\left[(-r)^{9} \alpha_{0}+(1-r)^{9} \alpha_{1}+(2-r)^{9} \alpha_{2}+\cdots+(7-r)^{9} \alpha_{7}+(8-r)^{9} \alpha_{8}\right] \\
-\frac{1}{10 !}\left[-r^{8} \beta_{0}+(1-r)^{8} \beta_{1}+(2-r)^{8} \beta_{2}+\cdots+(7-r)^{8} \beta_{7}+(8-r)^{8} \beta_{8}\right] & -\frac{1}{9 !}\left[-r^{9} \beta_{0}+(1-r)^{9} \beta_{1}+(2-r)^{9} \beta_{2}+\cdots+(7-r)^{9} \beta_{7}+(8-r)^{9} \beta_{8}\right] \\
D_{11}= & \frac{1}{11 !}\left[(-r)^{11} \alpha_{0}+(1-r)^{11} \alpha_{1}+(2-r)^{11} \alpha_{2}+\cdots++(7-r)^{11} \alpha_{7}+(8-r)^{11} \alpha_{8}\right] \\
D_{10}= & \frac{1}{10 !}\left[(-r)^{10} \alpha_{0}+(1-r)^{10} \alpha_{1}+(2-r)^{10} \alpha_{2}+\cdots+(7-r)^{10} \alpha_{7}+(8-r)^{10} \alpha_{8}\right] \\
& \\
& \left.=110 \beta_{1}+(2-r)^{10} \beta_{2}+\cdots++(7-r)^{10} \beta_{7}+(8-r)^{10} \beta_{8}\right]
\end{aligned}
$$

By setting $r=4$ in Equations (8) to (17), $D_{q}=0, q=2,3, \cdots \cdots 10$, and simplifying the resulting set of equations we obtain the following results.

$$
\begin{aligned}
\beta_{0}=\frac{23}{14175} a b c+\frac{52}{14175} a b+\frac{52}{14175} a c & +\frac{52}{14175} b c+\frac{188}{14175} a \\
& +\frac{188}{14175} b+\frac{188}{14175} c+\frac{3982}{14175}=\beta_{8} \\
\beta_{1}=-\frac{334}{14175} a b c-\frac{128}{2025} a b-\frac{128}{2025} a c & -\frac{128}{2025} b c-\frac{9844}{14175} a \\
& -\frac{9844}{14175} b+\frac{9844}{14175} c+\frac{23104}{14175}=\beta_{7} \\
\beta_{2}=\frac{2804}{14175} a b c+\frac{21976}{14175} a b+\frac{21976}{14175} a c+ & \frac{21976}{14175} b c-\frac{37936}{14175} a \\
& -\frac{37936}{14175} b-\frac{37936}{14175} c+\frac{7276}{14175}=\beta_{6}
\end{aligned}
$$




$$
\begin{aligned}
& \beta_{3}=-\frac{46378}{14175} a b c+\frac{70528}{14175} a b+\frac{70528}{14175} a c+ \frac{70528}{14175} b c-\frac{27268}{14175} a \\
&-\frac{27268}{14175} b-\frac{27268}{14175} c+\frac{77248}{14175}=\beta_{5} \\
& \beta_{4}=-\left[\beta_{0}+\beta_{1}+\beta_{2}+\beta_{3}+\beta_{5}+\beta_{6}+\beta_{7}+\beta_{8}\right]+8 \\
& 12(a+b+c)+8(1+2 a b+2 a c+2 b c)+4(a+b+c)
\end{aligned}
$$

And the expression for the error constant $D_{11}$ is

$$
D_{11}=\frac{2}{11 !}\left[-4^{11} \alpha_{0}-3^{11} \alpha_{1}-2^{11} \alpha_{2}-\alpha_{3}\right]-\frac{2}{10 !}\left[4^{10} \beta_{0}+3^{10} \beta_{1}+2^{10} \beta_{2}+\beta_{3}\right]
$$

The choice of values for the free parameters ${ }^{a, b}$ and $c$ is inspired by the desire to reduce the error constant as well as to derive a method that makes computation easier by decreasing the number of operations involved. The following are the preferable choices for the parameters $a_{,} b$ and $c$.

$$
a=\frac{1}{4}, \quad b=\frac{1}{12}, \quad c=-\frac{1}{3}
$$

and

$$
a=\frac{1}{2}, \quad b=\frac{2}{3}, \quad c=-\frac{1}{2}
$$

And the corresponding LMM schemes are

$$
\begin{aligned}
y_{n+8}+\frac{59}{36} y_{n+6} & +\frac{1}{18} y_{n+5}-\frac{1}{18} y_{n+3}-\frac{59}{36} y_{n+2}-y_{n}=h\left[\frac{190903}{680400} f_{n+8}^{(p)}+\frac{79499}{48600} f_{n+7}\right. \\
+\frac{63271}{170100} f_{n+6} & +\frac{1708871}{340200} f_{n+5}+\frac{2977}{68040} f_{n+4}+\frac{1708871}{340200} f_{n+3}+\frac{63277}{170100} f_{n+2} \\
+ & \frac{79499}{48600} f_{n+1}
\end{aligned}
$$

$$
\left.+\frac{190903}{680400} f_{n}\right]
$$

and

$$
\begin{aligned}
y_{n+8}-\frac{4}{3} y_{n+7}+ & y_{n+6}-y_{n+2}+\frac{4}{3} y_{n+1}-y_{n}=h\left[\frac{101}{350} f_{n+8}^{(p)}+\frac{89}{75} f_{n+7}-\frac{296}{175} f_{n+6}\right. \\
+ & \left.\frac{607}{175} f_{n+5}-\frac{263}{105} f_{n+4}+\frac{607}{175} f_{n+3}-\frac{296}{175} f_{n+2}+\frac{89}{75} f_{n+1}+\frac{101}{350} f_{n}\right]
\end{aligned}
$$

respectively.

The error constants for the derived schemes (27) and (28) are computed to be $-\frac{4309}{816480}$ and $-\frac{139}{23100}$ respectively.

\subsection{Convergence Analysis}

The first characteristic polynomial $\rho(\xi)$ of scheme (27) is

$$
\rho(\xi)=\xi^{8}+\frac{59}{36} \xi^{6}+\frac{1}{18} \xi^{5}-\frac{1}{18} \xi^{3}-\frac{59}{36} \xi^{2}-1
$$

From the first characteristic polynomial $\rho(\xi)$ of the scheme (27) we have 


$$
\begin{aligned}
& \rho(1)=\sum_{j=0}^{8} \alpha_{j}=1+\frac{59}{36}+\frac{1}{18}-\frac{1}{18}-\frac{59}{36}-1=0 \\
& \rho^{g}(1)=\sum_{j=0}^{8} j \alpha_{j}=8(1)+\frac{59}{6}(1)+\frac{5}{18}(1)-\frac{1}{6}(1)-\frac{59}{18}=\frac{44}{3}
\end{aligned}
$$

And the second characteristic polynomial $\sigma(\xi)$ of scheme (27) is

$$
\begin{aligned}
\sigma(\xi)=\frac{190903}{680400} \xi^{8}+\frac{79499}{48600} \xi^{7} & +\frac{63271}{170100} \xi^{6}+\frac{1708871}{340200} \xi^{5}+\frac{2977}{68040} \xi^{4}+\frac{1708871}{340200} \xi^{3} \\
& +\frac{63277}{170100} \xi^{2}+\frac{79499}{48600} \xi+\frac{190903}{680400}
\end{aligned}
$$

From Equation (32) we compute

$$
\sigma(1)=\frac{44}{3}
$$

From Equations (30), (31) and (32a) we observed that

$$
\left.\begin{array}{rl} 
& \text { (i) } \rho(1)=0 \\
3 & \text { (ii) } \rho^{v}(1)=\sigma(1)
\end{array}\right\}
$$

Therefore the scheme (27) is consistent.

Also, the roots of Equation (29) are computed as follows

$$
\left.\begin{array}{c}
\xi_{1}=1, \quad \xi_{2}=-1, \quad \xi_{3}=\frac{1}{4}-\frac{1}{4} \sqrt{15} i, \quad \xi_{4}=\frac{1}{4}+\frac{1}{4} \sqrt{15} i \\
\xi_{5}=-\frac{1}{3}-\frac{2}{3} \sqrt{2} i, \quad \xi_{6}=-\frac{1}{3}+\frac{2}{3} \sqrt{2} i \\
\xi_{7}=\frac{1}{12}-\frac{1}{12} \sqrt{143} i, \quad \xi_{8}=\frac{1}{12}+\frac{1}{12} \sqrt{143} i
\end{array}\right\}
$$

Each of the roots in Equations (34) have a modulus of 1. Thus the scheme (27) is also zero - stable. Hence, it is convergent.

Similarly, the first characteristic polynomial $\rho(\xi)$ of scheme $(28)$ is

$$
\rho(\xi)=\xi^{8}-\frac{4}{3} \xi^{7}+\xi^{6}-\xi^{2}+\frac{4}{3} \xi-1
$$

From Equation (35) we have

$$
\rho(1)=1-\frac{4}{3}+1-1+\frac{4}{3}-1=0
$$

and

$$
\rho^{\prime}(\xi)=8 \xi^{7}-\frac{28}{3} \xi^{6}+6 \xi^{5}-2 \xi+\frac{4}{3}=8-\frac{28}{3}+6-2+\frac{4}{3}=4
$$

And the second characteristic polynomial $\sigma(\xi)$ of scheme (28) is

$$
\begin{aligned}
\sigma(\xi)=\frac{101}{350} \xi^{8}+\frac{89}{75} \xi^{7}-\frac{296}{175} \xi^{6}+\frac{607}{175} \xi^{5} & -\frac{263}{105} \xi^{4}+\frac{607}{175} \xi^{3} \\
& -\frac{296}{175} \xi^{2}+\frac{89}{75} \xi+\frac{101}{350}
\end{aligned}
$$


From Equation (38) we compute

$$
\sigma(1)=4
$$

From Equations (36), (37) and (39) we observed that

$$
\left.\begin{array}{l}
\text { (i) } \rho(1)=0 \\
\text { (ii) } \rho^{\prime}(1)=\sigma(1)
\end{array}\right\}
$$

Therefore the scheme (28) is consistent.

Also, the roots of Equation (35) are computed as follows

$$
\left.\begin{array}{rl}
\xi_{1}=1, \quad \xi_{2} & =-1, \xi_{3}=-\frac{1}{2}-\frac{1}{2} \sqrt{3} i, \quad \xi_{4}=-\frac{1}{2}+\frac{1}{2} \sqrt{3} i \\
\xi_{5} & =\frac{2}{3}-\frac{1}{3} \sqrt{5} i, \quad \xi_{6}=\frac{2}{3}+\frac{1}{3} \sqrt{5} i \\
\xi_{7} & =\frac{1}{2}-\frac{1}{2} \sqrt{3} i, \quad \xi_{8}=\frac{1}{2}+\frac{1}{2} \sqrt{3} i
\end{array}\right\}
$$

Each of the roots in Equations (41) have a modulus of 1. Thus the scheme (28) is also zero - stable. Hence, it is convergent.

\subsection{Numerical Experiments}

The following scheme is an explicit tenth - order Adams -Bashforth method, due to [8].

$$
\begin{aligned}
y_{n+1}=y_{n}+\frac{h}{7257600} & {\left[49537553 f_{n}-259077637 f_{n-1}+805221248 f_{n-2}\right.} \\
-1533238912 f_{n-3}+ & 1886585258 f_{n-4}-1523349298 f_{n-5}+791906792 f_{n-6} \\
- & \left.248389768 f_{n-7}+401445117 f_{n-8}-2082753 f_{n-9}\right]
\end{aligned}
$$

Scheme (42) is used as a predictor for schemes (27) and (28) to form two sets of predictor - corrector pairs for the numerical solution of initial value problems. In order to generate the starting values for the predictor - corrector schemes, a tenth - order Runge - Kutta method due to Hairer [9] is employed.

The following problems are solved with the derived methods.

Problem 1: $y^{y}=x+y_{y} \quad y(0)=1, h=0.1, \quad 0 \leq x \leq 2$

Exact Solution: $Y_{E}(x)=2 e^{x}-1-x$

Problem 2: $y^{\prime}=7 x^{6}-10 x^{4}+9 x^{2}+2, y(0)=1, h=0.1,0 \leq x \leq 2$

Exact Solution: $Y_{E}(x)=x^{7}-2 x^{5}+3 x^{3}+2 x+1$

\section{RESULTS AND DISCUSSION}

A Tables 1 to 4 depict the results of applyin the predictor - corrector methods to some sample problems.

Table 1. Results of problem 1 with scheme (27)

\begin{tabular}{|l|l|r|l|}
\hline $\boldsymbol{x}$ & \multicolumn{1}{|c|}{ Exact solution } & Approximate & \multicolumn{1}{c|}{ Error } \\
\hline $\mathbf{0 . 0}$ & 1.0000000000 & 1.0000000000 & $0.0000000000 \mathrm{E}+00$ \\
\hline $\mathbf{0 . 1}$ & 1.1103418362 & 1.1103418362 & $4.8759885019 \mathrm{E}-11$ \\
\hline $\mathbf{0 . 2}$ & 1.2428055163 & 1.2428055163 & $2.0210055851 \mathrm{E}-11$ \\
\hline $\mathbf{0 . 3}$ & 1.3997176152 & 1.3997176152 & $4.8219872539 \mathrm{E}-11$ \\
\hline $\mathbf{0 . 4}$ & 1.5836493953 & 1.5836493953 & $1.7799983709 \mathrm{E}-11$ \\
\hline $\mathbf{0 . 5}$ & 1.7974425414 & 1.7974425414 & $2.2981616610 \mathrm{E}-13$ \\
\hline $\mathbf{0 . 6}$ & 2.0442376008 & 2.0442376008 & $1.9649615268 \mathrm{E}-11$ \\
\hline
\end{tabular}


Predictor - Corrector Methods of High Order for Numerical Integration of Initial Value Problems

\begin{tabular}{|l|l|r|l|}
\hline \hline $\mathbf{0 . 7}$ & 2.3275054149 & 2.3275054149 & $4.0069725316 \mathrm{E}-11$ \\
\hline $\mathbf{0 . 8}$ & 2.6510818570 & 2.6510818570 & $1.6209700249 \mathrm{E}-11$ \\
\hline $\mathbf{0 . 9}$ & 3.0192062223 & 3.0192062223 & $1.2440271036 \mathrm{E}-11$ \\
\hline $\mathbf{1 . 0}$ & 3.4365636569 & 3.4365636572 & $2.9999958073 \mathrm{E}-10$ \\
\hline $\mathbf{1 . 1}$ & 3.9083320479 & 3.9083320482 & $3.0000002482 \mathrm{E}-10$ \\
\hline $\mathbf{1 . 2}$ & 4.4402338455 & 4.4402338454 & $1.0000000827 \mathrm{E}-10$ \\
\hline $\mathbf{1 . 3}$ & 5.0385933352 & 5.0385933352 & $0.0000000000 \mathrm{E}+00$ \\
\hline $\mathbf{1 . 4}$ & 5.7103999337 & 5.7103999342 & $5.0000004137 \mathrm{E}-10$ \\
\hline $\mathbf{1 . 5}$ & 6.4633781407 & 6.4633781414 & $7.0000005792 \mathrm{E}-10$ \\
\hline $\mathbf{1 . 6}$ & 7.3060648488 & 7.3060648491 & $3.0000002482 \mathrm{E}-10$ \\
\hline $\mathbf{1 . 7}$ & 8.2478947835 & 8.2478947837 & $2.0000001655 \mathrm{E}-10$ \\
\hline $\mathbf{1 . 8}$ & 9.2992949288 & 9.2992949293 & $5.0000004137 \mathrm{E}-10$ \\
\hline $\mathbf{1 . 9}$ & 10.4717888846 & 10.4717888856 & $1.0000000827 \mathrm{E}-09$ \\
\hline $\mathbf{2 . 0}$ & 11.7781121979 & 11.7781121993 & $1.4000001158 \mathrm{E}-09$ \\
\hline
\end{tabular}

Table 2. Results of problem 1 with scheme (28)

\begin{tabular}{|l|l|r|l|}
\hline $\boldsymbol{x}$ & \multicolumn{1}{|c|}{ Exact solution } & Approximate & \multicolumn{1}{|c|}{ Error } \\
\hline $\mathbf{0 . 0}$ & 1.0000000000 & 1.0000000000 & $0.0000000000 \mathrm{E}+00$ \\
\hline $\mathbf{0 . 1}$ & 1.1103418362 & 1.1103418362 & $4.8759885019 \mathrm{E}-11$ \\
\hline $\mathbf{0 . 2}$ & 1.2428055163 & 1.2428055163 & $2.0210055851 \mathrm{E}-11$ \\
\hline $\mathbf{0 . 3}$ & 1.3997176152 & 1.3997176152 & $4.8219872539 \mathrm{E}-11$ \\
\hline $\mathbf{0 . 4}$ & 1.5836493953 & 1.5836493953 & $1.7799983709 \mathrm{E}-11$ \\
\hline $\mathbf{0 . 5}$ & 1.7974425414 & 1.7974425414 & $2.2981616610 \mathrm{E}-13$ \\
\hline $\mathbf{0 . 6}$ & 2.0442376008 & 2.0442376008 & $1.9649615268 \mathrm{E}-11$ \\
\hline $\mathbf{0 . 7}$ & 2.3275054149 & 2.3275054149 & $4.0069725316 \mathrm{E}-11$ \\
\hline $\mathbf{0 . 8}$ & 2.6510818570 & 2.6510818570 & $1.6209700249 \mathrm{E}-11$ \\
\hline $\mathbf{0 . 9}$ & 3.0192062223 & 3.0192062223 & $1.2440271036 \mathrm{E}-11$ \\
\hline $\mathbf{1 . 0}$ & 3.4365636569 & 3.4365636572 & $2.9999958073 \mathrm{E}-10$ \\
\hline $\mathbf{1 . 1}$ & 3.9083320479 & 3.9083320486 & $7.0000005792 \mathrm{E}-10$ \\
\hline $\mathbf{1 . 2}$ & 4.4402338455 & 4.4402338465 & $1.0000000827 \mathrm{E}-09$ \\
\hline $\mathbf{1 . 3}$ & 5.0385933352 & 5.0385933364 & $1.2000000993 \mathrm{E}-09$ \\
\hline $\mathbf{1 . 4}$ & 5.7103999337 & 5.7103999348 & $1.1000000910 \mathrm{E}-09$ \\
\hline $\mathbf{1 . 5}$ & 6.4633781407 & 6.4633781416 & $9.0000007447 \mathrm{E}-10$ \\
\hline $\mathbf{1 . 6}$ & 7.3060648488 & 7.3060648500 & $1.2000000993 \mathrm{E}-09$ \\
\hline $\mathbf{1 . 7}$ & 8.2478947835 & 8.2478947853 & $1.8000001489 \mathrm{E}-09$ \\
\hline $\mathbf{1 . 8}$ & 9.2992949288 & 9.2992949315 & $2.7000002234 \mathrm{E}-09$ \\
\hline $\mathbf{1 . 9}$ & 10.4717888846 & 10.4717888879 & $3.3000002730 \mathrm{E}-09$ \\
\hline $\mathbf{2 . 0}$ & 11.7781121979 & 11.7781122016 & $3.7000003061 \mathrm{E}-09$ \\
\hline
\end{tabular}

Table 3. Results of problem 2 with scheme (27)

\begin{tabular}{|l|l|r|l|}
\hline $\boldsymbol{x}$ & \multicolumn{1}{|c|}{ Exact solution } & Approximate & \multicolumn{1}{c|}{ Error } \\
\hline $\mathbf{0 . 0}$ & 1.0000000000 & 1.0000000000 & $0.0000000000 \mathrm{E}+00$ \\
\hline $\mathbf{0 . 1}$ & 1.2029801000 & 1.2029801000 & $0.0000000000 \mathrm{E}+00$ \\
\hline $\mathbf{0 . 2}$ & 1.4233728000 & 1.4233728000 & $0.0000000000 \mathrm{E}+00$ \\
\hline $\mathbf{0 . 3}$ & 1.6763587000 & 1.6763587000 & $0.0000000000 \mathrm{E}+00$ \\
\hline $\mathbf{0 . 4}$ & 1.9731584000 & 1.9731584000 & $0.0000000000 \mathrm{E}+00$ \\
\hline $\mathbf{0 . 5}$ & 2.3203125000 & 2.3203125000 & $0.0000000000 \mathrm{E}+00$ \\
\hline $\mathbf{0 . 6}$ & 2.7204736000 & 2.7204736000 & $0.0000000000 \mathrm{E}+00$ \\
\hline $\mathbf{0 . 7}$ & 3.1752143000 & 3.1752143000 & $0.0000000000 \mathrm{E}+00$ \\
\hline $\mathbf{0 . 8}$ & 3.6903552000 & 3.6903552000 & $0.0000000000 \mathrm{E}+00$ \\
\hline
\end{tabular}


Abdulrahman Ndanusa \& Fatima Umar Tafida

\begin{tabular}{|l|l|r|l|}
\hline \hline $\mathbf{0 . 9}$ & 4.2843169000 & 4.2843169000 & $0.0000000000 \mathrm{E}+00$ \\
\hline $\mathbf{1 . 0}$ & 5.0000000000 & 5.0000000000 & $0.0000000000 \mathrm{E}+00$ \\
\hline $\mathbf{1 . 1}$ & 5.9206971000 & 5.9206971000 & $0.0000000000 \mathrm{E}+00$ \\
\hline $\mathbf{1 . 2}$ & 7.1905408000 & 7.1905408000 & $0.0000000000 \mathrm{E}+00$ \\
\hline $\mathbf{1 . 3}$ & 9.0399917000 & 9.0399917000 & $0.00000000000 \mathrm{E}+00$ \\
\hline $\mathbf{1 . 4}$ & 11.8168704000 & 11.8168704000 & $0.0000000000 \mathrm{E}+00$ \\
\hline $\mathbf{1 . 5}$ & 16.0234375000 & 16.0234375000 & $0.0000000000 \mathrm{E}+00$ \\
\hline $\mathbf{1 . 6}$ & 22.3600256000 & 22.3600256000 & $0.0000000000 \mathrm{E}+00$ \\
\hline $\mathbf{1 . 7}$ & 31.7757273000 & 31.7757273000 & $0.00000000000 \mathrm{E}+00$ \\
\hline $\mathbf{1 . 8}$ & 45.5266432000 & 45.5266432000 & $0.0000000000 \mathrm{E}+00$ \\
\hline $\mathbf{1 . 9}$ & 65.2421939000 & 65.2421939000 & $0.0000000000 \mathrm{E}+00$ \\
\hline $\mathbf{2 . 0}$ & 93.0000000000 & 93.0000000000 & $0.0000000000 \mathrm{E}+00$ \\
\hline
\end{tabular}

Table 4. Results of problem 2 with scheme (28)

\begin{tabular}{|c|l|r|l|}
\hline $\boldsymbol{x}$ & \multicolumn{1}{|c|}{ Exact solution } & Approximate & \multicolumn{1}{c|}{ Error } \\
\hline $\mathbf{0 . 0}$ & 1.0000000000 & 1.0000000000 & $0.0000000000 \mathrm{E}+00$ \\
\hline $\mathbf{0 . 1}$ & 1.2029801000 & 1.2029801000 & $0.0000000000 \mathrm{E}+00$ \\
\hline $\mathbf{0 . 2}$ & 1.4233728000 & 1.4233728000 & $0.0000000000 \mathrm{E}+00$ \\
\hline $\mathbf{0 . 3}$ & 1.6763587000 & 1.6763587000 & $0.0000000000 \mathrm{E}+00$ \\
\hline $\mathbf{0 . 4}$ & 1.9731584000 & 1.9731584000 & $0.0000000000 \mathrm{E}+00$ \\
\hline $\mathbf{0 . 5}$ & 2.3203125000 & 2.3203125000 & $0.0000000000 \mathrm{E}+00$ \\
\hline $\mathbf{0 . 6}$ & 2.7204736000 & 2.7204736000 & $0.0000000000 \mathrm{E}+00$ \\
\hline $\mathbf{0 . 7}$ & 3.1752143000 & 3.1752143000 & $0.0000000000 \mathrm{E}+00$ \\
\hline $\mathbf{0 . 8}$ & 3.6903552000 & 3.6903552000 & $0.0000000000 \mathrm{E}+00$ \\
\hline $\mathbf{0 . 9}$ & 4.2843169000 & 4.2843169000 & $0.0000000000 \mathrm{E}+00$ \\
\hline $\mathbf{1 . 0}$ & 5.0000000000 & 5.0000000000 & $0.0000000000 \mathrm{E}+00$ \\
\hline $\mathbf{1 . 1}$ & 5.9206971000 & 5.9206971000 & $0.0000000000 \mathrm{E}+00$ \\
\hline $\mathbf{1 . 2}$ & 7.1905408000 & 7.1905408000 & $0.0000000000 \mathrm{E}+00$ \\
\hline $\mathbf{1 . 3}$ & 9.0399917000 & 9.0399917000 & $0.0000000000 \mathrm{E}+00$ \\
\hline $\mathbf{1 . 4}$ & 11.8168704000 & 11.8168704000 & $0.0000000000 \mathrm{E}+00$ \\
\hline $\mathbf{1 . 5}$ & 16.0234375000 & 16.0234375000 & $0.0000000000 \mathrm{E}+00$ \\
\hline $\mathbf{1 . 6}$ & 22.3600256000 & 22.3600256000 & $0.0000000000 \mathrm{E}+00$ \\
\hline $\mathbf{1 . 7}$ & 31.7757273000 & 31.7757273000 & $0.0000000000 \mathrm{E}+00$ \\
\hline $\mathbf{1 . 8}$ & 45.5266432000 & 45.5266432000 & $0.0000000000 \mathrm{E}+00$ \\
\hline $\mathbf{1 . 9}$ & 65.2421939000 & 65.2421939000 & $0.0000000000 \mathrm{E}+00$ \\
\hline $\mathbf{2 . 0}$ & 93.0000000000 & 93.0000000000 & $0.0000000000 \mathrm{E}+00$ \\
\hline
\end{tabular}

In Table 1, Scheme (27) exhibited a steady rise in errors as the step increases for the first few steps i.e., from $x=0.0$ to $x=0.4$; and at $x=0.5$ it has $2.2981616610 E-13$, while it goes back to the same trend from $x=0.6$ to $x=0.9$. Thereafter, from $x=1.0$ to $x=1.2$ it shows a steady growth. At $x=1.3$, it has $0.0000000000 E+00$. And from $x=1.4$ to $x=1.8$ it maintains the same trend as in $=1.0$ to $x=1.2$. Then it decreases between $x=1.9$ and $x=2.0$. Generally, the scheme exhibited minimal error.

In Table 2, Scheme (28) demonstrated a similar trend as in Table 1. At $x=0.5$ the error is $2.2981616610 E-13$; while at $x=1.0$ the error is $2.9999958073 E-10$. Finally at $x=2.0$ the error stands at $3.7000003061 E-09$.

In Tables 3 and 4, the two schemes solved the differential equation exactly, with no error at all. This is expected, as the exact solution of problem 2 is a polynomial of degree 6 , i.e., its degree is less than 8 , which is the step length of the methods. 


\section{Conclusion}

Two implicit LMMs of order ten are derived and combined with an explicit method of the same order to form predictor - corrector pairs. The derived schemes have proven to be effective and efficient by satisfying convergence criteria as well as solving some sample differential equation problems correctly.

\section{REFERENCES}

[1] J.D. Lambert, Numerical Methods for Ordinary Differential Systems: The Initial Value Problem, New York, USA. John Wiley \& Sons Ltd., 1991, ch. 4, pp. 103.

[2] Milne, W. E., Numerical integration of ordinary differential equations, American Mathematical Monthly. 33, 455 - 460 (1926).

[3] Hamming, R. W., Stable predictor - corrector methods for ordinary differential equations, Journal of Association of Computing Machinery. 6, 37 - 47 (1959).

[4] Klopfenstein, R. W. and Millman, R. S., Numerical stability of a one evaluation predictor corrector algorithm for numerical solution of ordinary differential equations, Mathematics of Computation. 22, $557-564$ (1968).

[5] Crane, R. L. and Klopfenstein, R. W., A predictor - corrector algorithm with an increased range of absolute stability, Journal of Association for Computing Machinery. 12, 227 - 241 (1965).

[6] Krogh, F. T., Predictor - corrector methods of high order with improved stability characteristics, Journal of Association for Computing Machinery. 13, 374 - 385 (1966).

[7] Ndanusa, A. and Adeboye, K. R., An optimal 6-step implicit linear multistep method for initial value problems, Journal of Research in Physical Sciences. 4(1), 93 - 99 (2008).

[8] Tafida, F. U., A tenth - order predictor - corrector method for numerical integration of initial value problems, Unpublished Master's Thesis, Federal Uinversity of Technology, Minna, Nigeria. Pp. 47 (2015).

[9] Hairer, E., A Runge - Kutta method of order 10, Journal of Institute of Mathematics and its. Applications. 21, 47-59 (1978).

\section{AUTHORS' BIOGRAPHY}

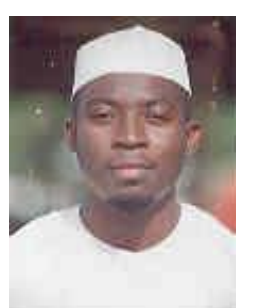

Dr Abdulrahman Ndanusa, obtained a PhD in Applied Mathematics from Federal University of Technology, Minna, Nigeria in 2012. He is a Senior Lecturer in the Department of Mathematics at Federal University of Technology, Minna, Nigeria where he teaches various courses to undergraduate and postgraduate students. He is an active researcher in Numerical Analysis and Biomathematics where he has several publications in international peer- reviewed journals.

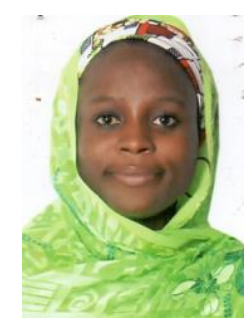

Fatima Umar Tafida, received a BTech in Mathematics with Computer science (in 2008), and an MTech in Mathematics (in 2014), from Federal University of Technology, Minna. She is at present an ICT officer at Ibrahim Badamasi Babangida University, Lapai, Nigeria. 